\title{
Exploiting Passive Dynamics with Variable Stiffness Actuation in Robot Brachiation
}

\author{
Jun Nakanishi and Sethu Vijayakumar \\ School of Informatics, University of Edinburgh, United Kingdom \\ Email: jun.nakanishi@ed.ac.uk, sethu.vijayakumar@ed.ac.uk
}

\begin{abstract}
This paper explores a passive control strategy with variable stiffness actuation for swing movements. We consider brachiation as an example of a highly dynamic task which requires exploitation of gravity in an efficient manner for successful task execution. First, we present our passive control strategy considering a pendulum with variable stiffness actuation. Then, we formulate the problem based an optimal control framework with temporal optimization in order to simultaneously find an appropriate stiffness profile and movement duration such that the resultant movement will be able to exploit the passive dynamics of the robot. Finally, numerical evaluations on a twolink brachiating robot with a variable stiffness actuator (VSA) model are provided to demonstrate the effectiveness of our approach under different task requirements, modelling errors and switching in the robot dynamics. In addition, we discuss the issue of task description in terms of the choice of cost function for successful task execution in optimal control.
\end{abstract}

\section{INTRODUCTION}

In recent years, there has been growing effort in the development of variable stiffness actuators. Various designs of actuators with mechanically adjustable stiffness/compliance composed of passive elastic elements such as springs have been proposed [4, 6, 11, 12, 14, 15]. In contrast to conventional stiff actuators, one of the motivations to develop variable stiffness actuators is that such actuators are expected to have desirable properties such as compliant actuation, energy storage capability with potential applications in human-robot interaction and improvements of task performance in dynamic tasks.

This paper explores a control strategy for exploiting passive dynamics in tasks involving swing movements with variable stiffness actuation based on optimal control. Despite potential benefits of variable stiffness joints, finding an appropriate control strategy to fully exploit the capabilities of variable stiffness actuators (VSAs) is challenging due to the increased complexity of mechanical properties and the number of control variables. Taking an optimal control approach, recent studies in [3, 8, 10] have investigated the benefits of variable stiffness actuation such as energy storage in explosive movements from a viewpoint of performance improvement. Braun et al. [3] have demonstrated such benefits of VSAs by simultaneously optimizing time-varying torque and stiffness profiles of the actuator in a ball throwing task. In [8, 10], an optimal control problem of maximizing link velocity with variable stiffness actuator models has been investigated. It is shown that much larger link velocity can be achieved than that of the motor in the VSA with the help of appropriate stiffness adjustment during a hitting movement. In a similar problem, Hondo and Mizuuchi [13] have discussed the issue of determining the inertia parameter and spring constant in the design of series elastic actuators to increase the peak velocity. In robot running, Karssen and Wisse [16] have presented numerical studies to demonstrate that an optimized nonlinear leg stiffness profile could improve robustness against disturbances.

In this paper, we focus on the passive control strategy with variable stiffness actuation for swing movements in a brachiation task. Indeed, the importance of exploitation of the intrinsic passive dynamics for efficient actuation and control has been discussed in the study of passive dynamic walking where biped robots with no actuation or minimal actuation can exhibit human-like natural walking behavior [5]. In this study, we consider brachiation as an example of dynamic task involving swing movement. Brachiation is an interesting form of locomotion of an ape swinging from handhold to handhold like a pendulum [7, 29] which requires explicit exploitation of the passive dynamics with the help of gravity to achieve the task. From a control point of view, designing a brachiating controller is a challenging problem since the system is underactuated, i.e., there is no actuation at the gripper. Efforts have been made to develop a control law for a class of underactuated systems from a control theoretic view 1 , e.g., [18, 19, 22, 32].

In our previous study [23], we have proposed a method of describing the task using a dynamical system based on a nonlinear control approach, and derived a nonlinear control law for a joint torque controlled two-link brachiating robot. The control strategy in [23] uses an active cancellation of the plant dynamics using input-output linearization to force the robot to mimic the specified pendulum-like motion described in terms of target dynamics. In contrast, Gomes and Ruina [9] studied brachiation with zero-energy-cost motions using only passive dynamics of the body. They sought numerical solutions for the initial conditions which lead to periodically continuous locomotion without any joint torques. By extending the (unstable) fixed point solutions in unactuated horizontal brachiation found in [9], Rosa et al. [28] numerically studied open-loop stable (unactuated downhill and powered uphill) brachiation of a two-link model from a viewpoint of hybrid systems control including switching and discontinuous transitions.

\footnotetext{
${ }^{1}$ Much of the related work has focused on the motion planning of underactuated manipulators in a horizontal plane (not necessarily under the influence of the gravity). In such a case, dynamic coupling of link inertia is exploited rather than the passive dynamics due to gravity.
} 
Motivated by the work in [9], our goal in this study is to demonstrate that highly dynamic tasks such as brachiation can be achieved by fully exploiting passive dynamics with simultaneous stiffness and temporal optimization. In our recent work [24], effectiveness of temporal optimization and stiffness optimization in periodic movements has been discussed. However, temporal optimization and stiffness optimization are treated separately and a rather simplified, ideal actuator models were used in the evaluation. In this study, numerical evaluations of our approach on a two-link brachiating robot with a realistic MACCEPA (Mechanically Adjustable Compliance and Controllable Equilibrium Position Actuator) VSA model [11] (see motivation for this particular VSA in Section III-B) are provided to show the effectiveness of our approach under different task requirements, modelling errors and variations of the robot dynamics. Furthermore, we also discuss the issue of and effect of task encoding via an appropriate choice of the cost function for successful task execution.

\section{Passive Control Strategy in Swing Movement WITH VARIABLE STIFFNESS ACTUATION}

Our goal in this paper is to devise a control strategy to achieve the desired swing maneuver in brachiation by exploiting natural dynamics of the system. To begin with, we discuss our approaches of implementing a passive control strategy, considering a pendulum with variable stiffness actuation.

A natural and desirable strategy would be to make good use of gravity by making the joints passive and compliant. For example, in walking, unlike many high gain position controlled biped robots with stiff joints, humans seem to let the lower leg swing freely by relaxing the muscles controlling the knee joint during the swing phase and increase stiffness only when necessary. In fact, stiffness modulation is observed during a walking cycle in a cat locomotion study 2 [1].

Consider the dynamics of a simplified single-link pendulum under the influence of gravity. If we consider an idealized VSA model of the form $\tau=-k\left(q-q_{m}\right)$, where $q$ is the joint angle, $\tau$ is the joint torque, $k$ is the stiffness and $q_{m}$ is the equilibrium position of the elastic actuator, then the dynamics can be written as:

$$
m l^{2} \ddot{q}+m g l \sin q=\tau=-k\left(q-q_{m}\right)
$$

where $m$ is the mass, $l$ is the length and $g$ is the gravitational constant. In this idealized VSA model, we assume that $k$ and $q_{m}$ are the control variables. From a viewpoint of position control, one way of looking at this system is as a manipulator with a flexible (elastic) joint, where we solve a tracking control problem [31]. Recently, Palli et al. [25], proposed a tracking control algorithm for such a flexible joint manipulator with variable stiffness actuation to achieve asymptotic tracking to the desired joint and stiffness trajectories based on inputoutput linearization, effectively an active cancellation of the intrinsic robot dynamics. Note that the main focus of [25] is

\footnotetext{
${ }^{2}$ To our knowledge, there are a large number of studies of stretch reflexes modulation in human walking, however, something that specifically addresses stiffness modulation is very limited. In human arm cyclic movement, Bennett et al. [2] reported time-varying stiffness modulation in the elbow joint.
}

the tracking control of the given joint and stiffness trajectories, and the problem of generating such desired trajectories for a given specific task is not addressed.

On the other hand, if we rearrange the linearized dynamics of (1) $(\sin q \approx q)$ as

$$
m l^{2} \ddot{q}+(m g l+k) q=v
$$

where $v=k q_{m}$, another view of the control problem could be that varying the stiffness of the actuator $k$ in the second term of the left hand side effectively changes the dynamics property, e.g., the natural frequency of the pendulum. From this perspective, the control problem can be framed as finding an appropriate (preferably small) stiffness profile $k$ to modulate the system dynamics (only when necessary) and compute the virtual equilibrium trajectory $q_{m}$ [30] to fulfill the specified task requirement while maximally exploiting the natural dynamics.

In a realistic situation, it is not straightforward to compute a control command for the actuator to realize such an idea due to the complexity of the system dynamics, actuator mechanisms, the requirement of coordination of multiple degrees of freedom and redundancy in actuation. Next, we exploit the framework of optimal control and spatiotemporal optimization of variable stiffness actuation to find appropriate control commands to implement the brachiation task.

\section{PROBLEM Formulation}

\section{A. Robot Dynamics}

The equation of motion of the two-link brachiating robot shown in Fig. 1 takes the standard form of rigid body dynamics where only the second joint has actuation:

$$
\mathbf{M}(\mathbf{q}) \ddot{\mathbf{q}}+\mathbf{C}(\mathbf{q}, \dot{\mathbf{q}}) \dot{\mathbf{q}}+\mathbf{g}(\mathbf{q})+\mathbf{D} \dot{\mathbf{q}}=\left[\begin{array}{l}
0 \\
\tau
\end{array}\right]
$$

where $\mathbf{q}=\left[\begin{array}{ll}q_{1}, & q_{2}\end{array}\right]^{T}$ is the joint angle vector, $\mathbf{M}$ is the inertia matrix, $\mathbf{C}$ is the Coriolis term, $\mathbf{g}$ is the gravity vector, $\mathbf{D}$ is the viscous damping matrix, and $\tau$ is the joint torque acting on the second joint.

\section{B. Variable Stiffness Actuation}

We consider a MACCEPA model [11] as our VSA implementation of choice. MACCEPA is one of the designs of mechanically adjustable compliant actuators with a passive elastic element (see Fig. 11). This actuator design has the desirable characteristics that the joint can be very compliant and mechanically passive/back-drivable: this allows free swinging with a large range of movement by relaxing the spring, highly suitable for the brachiation task we consider. MACCEPA is equipped with two position controlled servo motors, $q_{m 1}$ and $q_{m 2}$, each of which controls the equilibrium position and the spring pre-tension, respectively. The parameters of the robot we use in this study (Fig. 11(c)) are based on a 2-link MACCEPA joint (Fig. 1,b)) constructed in our lab [3].

The joint torque for this actuator model is given by

$\tau=\kappa \sin \left(q_{m 1}-q\right) B C\left(1+\frac{r_{d} q_{m 2}-(C-B)}{\sqrt{B^{2}+C^{2}-2 B C \cos \left(q_{m 1}-q\right)}}\right)$ 




(a) Brachiating robot model with VSA



(b) 2-link MACCEPA joint

\begin{tabular}{|c|c|c|c|}
\hline \multicolumn{2}{|c|}{ Robot parameters } & $\mathrm{i}=1$ & $\mathrm{i}=2$ \\
\hline Mass & $m_{i}(\mathrm{~kg})$ & 0.42 & 0.23 \\
\hline Moment of inertia & $I_{i}\left(\mathrm{kgm}^{2}\right)$ & 0.0022 & 0.0017 \\
\hline Link length & $l_{i}(\mathrm{~m})$ & 0.25 & 0.25 \\
\hline COM location & $l_{c i}(\mathrm{~m})$ & 0.135 & 0.0983 \\
\hline Viscous friction & $d_{i}(\mathrm{Nm} / \mathrm{s})$ & 0.01 & 0.01 \\
\hline
\end{tabular}

\begin{tabular}{|c|c|c|}
\hline \multicolumn{2}{|c|}{ MACCEPA parameters } & value \\
\hline Spring constant & $\kappa(\mathrm{N} / \mathrm{m})$ & 771 \\
\hline Lever length & $B(\mathrm{~m})$ & 0.03 \\
\hline Pin displacement & $C(\mathrm{~m})$ & 0.0125 \\
\hline Drum radius & $r_{d}(\mathrm{~m})$ & 0.01 \\
\hline
\end{tabular}

(c) Model parameters

Fig. 1. Model of a two-link brachiating robot with a MACCEPA variable stiffness actuator.

and the joint stiffness can be computed as

$$
k=-\frac{\partial \tau}{\partial q}=\kappa \cos (\alpha) B C\left(1+\frac{\beta}{\gamma}\right)-\frac{\kappa \sin ^{2}(\alpha) B^{2} C^{2} \beta}{\gamma^{3}}
$$

where $\kappa$ is the spring constant, $r_{d}$ is the drum radius, $\alpha=q_{m 1}-q, \beta=r_{d} q_{m 2}-(C-B)$ and $\gamma=$ $\sqrt{B^{2}+C^{2}-2 B C \cos \left(q_{m 1}-q\right)}$ (see Fig. 11a) and (c) for the definition of the model parameters and variables).

The spring tension is given by

$$
F=\kappa\left(l-l_{0}\right)
$$

where $l=\sqrt{B^{2}+C^{2}-2 B C \cos \left(q_{m 1}-q\right)}+r_{d} q_{m 2}$ is the current spring length and $l_{0}=C-B$ is the spring length at rest. The joint torque equation (4) also can be rearranged in terms of the moment arm and the spring tension as

$$
\tau=\frac{B C \sin \left(q_{m 1}-q\right)}{\gamma} F .
$$

Note that MACCEPA has a relatively simple configuration in terms of actuator design compared to other VSAs, however, the torque and stiffness relationships in (4) and (5) are dependent on the current joint angle and two servo motor angles in a complicated manner and its control is not straightforward.

In addition, we include realistic position controlled servo motor dynamics, approximated by a second order system with a PD feedback control

$$
\ddot{q}_{m i}+2 a_{i} \dot{q}_{m i}+a_{i}^{2}\left(q_{m i}-u_{i}\right)=0, \quad(i=1,2)
$$

where $u_{i}$ is the motor position command, $a_{i}$ determines the bandwidth of the actuator and the range of the servo motors are limited as $q_{m i, m i n} \leq q_{m i} \leq q_{m i, \max }$ and $u_{i, m i n} \leq u_{i} \leq$ $u_{i, \max }$ [3]. In this study, we use $a_{i}=50$.

\section{Augmented Plant Dynamics}

The plant dynamics composed of the robot dynamics (3) and the servo motor dynamics (8) can now be formulated as

$$
\dot{\mathbf{x}}=\mathbf{f}(\mathbf{x}, \mathbf{u})
$$

where

$$
\mathbf{f}=\left[\begin{array}{c}
\mathbf{x}_{2} \\
\mathbf{M}^{-1}\left(\mathbf{x}_{1}\right)\left(-\mathbf{C}\left(\mathbf{x}_{1}, \mathbf{x}_{2}\right) \mathbf{x}_{2}-\mathbf{g}\left(\mathbf{x}_{1}\right)-\mathbf{D} \dot{\mathbf{x}}_{2}+\left[\begin{array}{c}
0 \\
\tau\left(\mathbf{x}_{1}, \mathbf{x}_{3}\right)
\end{array}\right]\right) \\
\mathbf{x}_{4} \\
-\mathbf{a}^{2} \mathbf{x}_{3}-2 \mathbf{a x}_{4}+\mathbf{a}^{2} \mathbf{u}
\end{array}\right]
$$

$\mathbf{x}=\left[\begin{array}{llll}\mathbf{x}_{1}, & \mathbf{x}_{2}, & \mathbf{x}_{3}, & \mathbf{x}_{4}\end{array}\right]^{T}=\left[\mathbf{q}, \dot{\mathbf{q}}, \mathbf{q}_{m}, \dot{\mathbf{q}}_{m}\right]^{T} \in \mathbb{R}^{8}$, $\mathbf{q}=\left[q_{1}, q_{2}\right]^{T}, \mathbf{q}_{m}=\left[q_{m 1}, q_{m 2}\right]^{T}$ and $\mathbf{u}=\left[u_{1}, u_{2}\right]^{T}$. Note that $\mathbf{a}$ in (10) denotes $\mathbf{a}=\operatorname{diag}\left\{a_{i}\right\}$ and $\mathbf{a}^{2}$ is defined as $\mathbf{a}^{2}=\operatorname{diag}\left\{a_{i}^{2}\right\}$ for notational convenience.

\section{Optimal Feedback Control with Temporal Optimization}

For plant dynamics

$$
\dot{\mathbf{x}}=\mathbf{f}(\mathbf{x}, \mathbf{u}),
$$

the objective of optimal control [33] is to find a control law

$$
\mathbf{u}^{*}=\mathbf{u}(\mathbf{x}, t)
$$

which minimizes the cost function

$$
J=\Phi(\mathbf{x}(T))+\int_{0}^{T} h(\mathbf{x}(t), \mathbf{u}(t)) d t
$$

for a given movement duration $T$, where $\Phi(\mathbf{x}(T))$ is the terminal cost and $h(\mathbf{x}(t), \mathbf{u}(t))$ is the running cost. We employ the iterative linear quadratic Gaussian (ILQG) algorithm [17] to obtain a locally optimal feedback control law

$$
\mathbf{u}(\mathbf{x}, t)=\mathbf{u}^{o p t}(t)+\mathbf{L}(t)\left(\mathbf{x}(t)-\mathbf{x}^{o p t}(t)\right) .
$$

In addition to obtaining an optimal control law, we simultaneously optimize the movement duration $T$ using the temporal optimization algorithm proposed in [27]. In [27], a mapping $\beta(t)$ from the real time $t$ to a canonical time $t^{\prime}$

$$
t^{\prime}=\int_{0}^{t} \frac{1}{\beta(s)} d s
$$

is introduced and $\beta(t)$ is optimized to yield the optimal movement duration $T$. In this study, we simplify the temporal 
optimization algorithm by discretizing (15) with an assumption that $\beta(t)$ is constant during the movement as

$$
\Delta t^{\prime}=\frac{1}{\beta} \Delta t .
$$

By updating $\beta$ using gradient descent

$$
\beta_{\text {new }}=\beta-\eta \nabla_{\beta} J
$$

where $\eta>0$ is a learning rate, we obtain the movement duration $T^{\prime}=\frac{1}{\beta} T$ where $T=N \Delta t$ ( $N$ is the number of discrete time steps). In the complete optimization procedure, ILQG and the update of $\beta$ in (17) are iterated in an EM (ExpectationMaximization)-like manner until convergence to obtain the final optimal feedback control law (14) and the associated movement duration $T^{*}$. Depending on the task objective, it is further possible to augment the cost by including the time explicitly as

$$
J^{\prime}=J+w_{T} T
$$

where $J$ is the cost (13) and $w_{T}$ is the weight on the time cost, which determines trade-off between the original cost $J$ and movement duration $T$.

\section{Evaluations 3}

\section{A. Optimization Results in Brachiation Task}

In this paper, we consider the task of swing locomotion from handhold to handhold on a ladder. and swinging-up from the suspended posture to catch the target bar. Motivated by the discussions on our passive control strategy in Section [II, we consider the following cost function to encode the task (the specific reason will be explained below)

$J=\left(\mathbf{y}(T)-\mathbf{y}^{*}\right)^{T} \mathbf{Q}_{T}\left(\mathbf{y}(T)-\mathbf{y}^{*}\right)+\int_{0}^{T}\left(\mathbf{u}^{T} \mathbf{R}_{1} \mathbf{u}+R_{2} F^{2}\right) d t$

where $\mathbf{y}=[\mathbf{r}, \dot{\mathbf{r}}]^{T} \in \mathbb{R}^{4}$ are the position and the velocity of the gripper in the Cartesian coordinates, $\mathbf{y}^{*}$ is the target values when reaching the target $\mathbf{y}^{*}=\left[\mathbf{r}^{*}, \mathbf{0}\right]^{T}$ and $F$ is the spring tension in the VSA given in (6). This objective function is designed in order to reach the target located at $\mathbf{r}^{*}$ at the specified time $T$ while minimizing the spring tension $F$ in the VSA. Note that the main component in the running cost is to minimize the spring tension $F$ by the second term while the first term $\mathbf{u}^{T} \mathbf{R}_{1} \mathbf{u}$ is added for regularization with a small choice of the weights in $\mathbf{R}_{1}$. In practice, this is necessary since $F$ is a function of the state and ILQG requires a control cost in its formulation to compute the optimal control law.

Notice that the actuator torque (7) can be expressed in the form

$$
\tau=-F \sin \left(q-q_{m 1}\right) / \gamma^{\prime}
$$

where $\gamma^{\prime}=\sqrt{B^{2}+C^{2}-2 B C \cos \left(q_{m 1}-q\right)} / B C$. In this equation (20), it can be conceived that $F$ has a similar role to the stiffness parameter $k$ as in the simplified actuator model

$$
\tau=-k\left(q-q_{m}\right) .
$$

\footnotetext{
${ }^{3} \mathrm{~A}$ video clip of summarizing the results is available at http://goo.gl/iYrFr
}

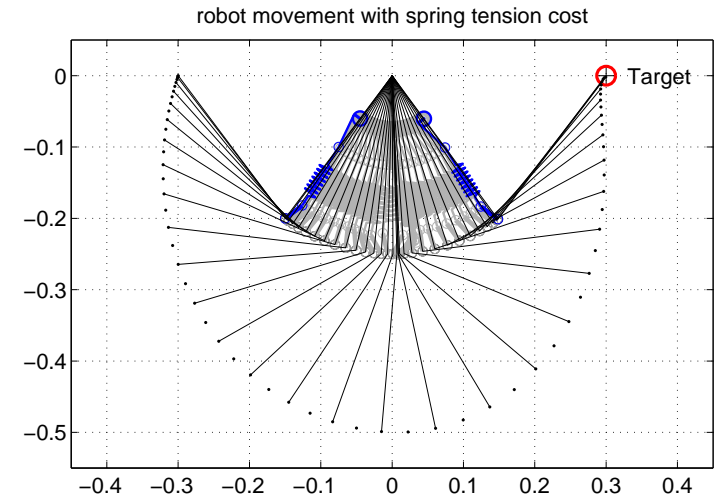

(a) Movement of the robot with optimized duration $T=0.606$ (sec) joint angles
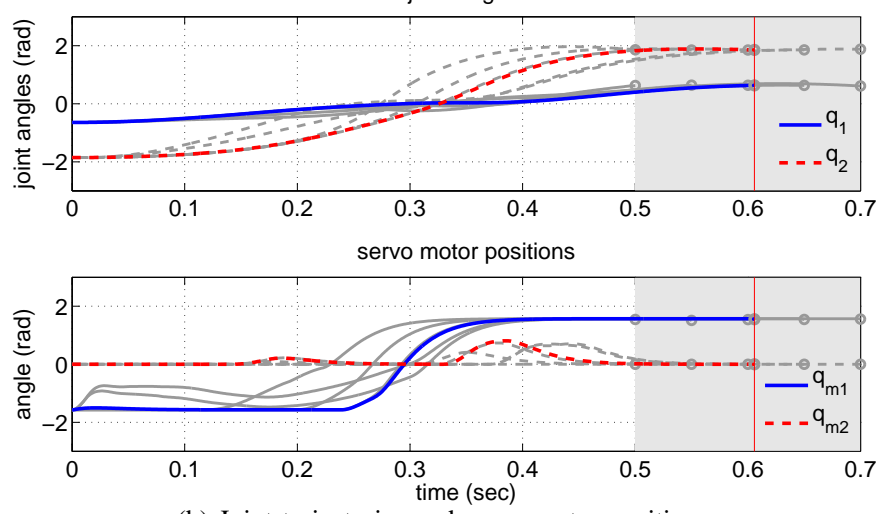

(b) Joint trajectories and servo motor positions
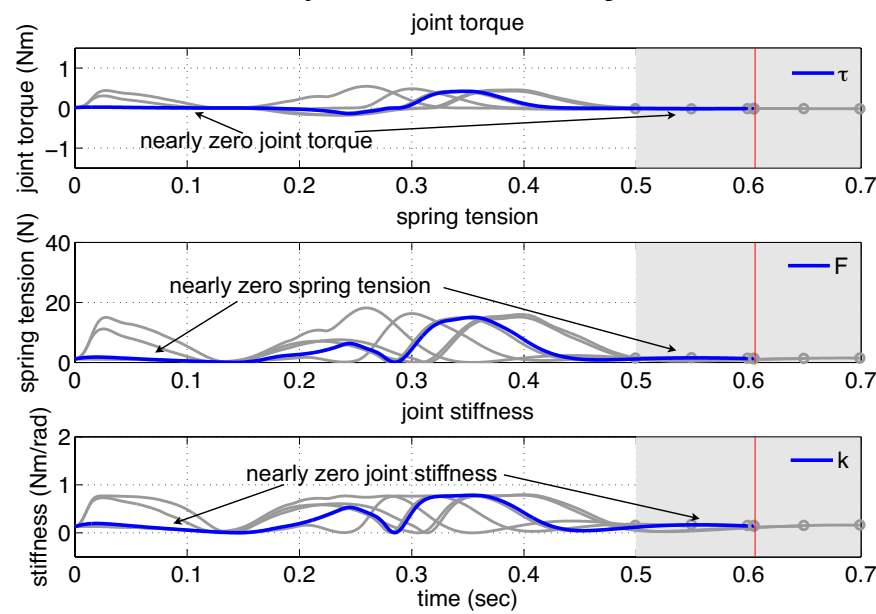

(c) Joint torque, spring tension and joint stiffness

Fig. 2. Optimization of the locomotion task using the cost 19]. In (b) and (c), gray thin lines show the plots for non-optimized $T$ in the range of $T=$ $[0.5, \cdots, 0.7](\mathrm{sec})$ and blue thick lines show the plots for optimized $T=$ 0.606 . Note that especially at the beginning and the end of the movement, joint torque, spring tension and joint stiffness are kept small allowing the joint to swing passively.

Another interpretation can be considered in such a way that if we linearize (4) around the equilibrium position assuming that $\alpha=q_{m 1}-q \ll 1$, the relationship between the joint stiffness $k$ in (5) and the spring tension $F$ in (6) can be approximated as

$$
k \approx \frac{1}{\sqrt{B^{2}+C^{2}-2 B C}} F .
$$

Thus, effectively, minimizing the spring tension $F$ corresponds 


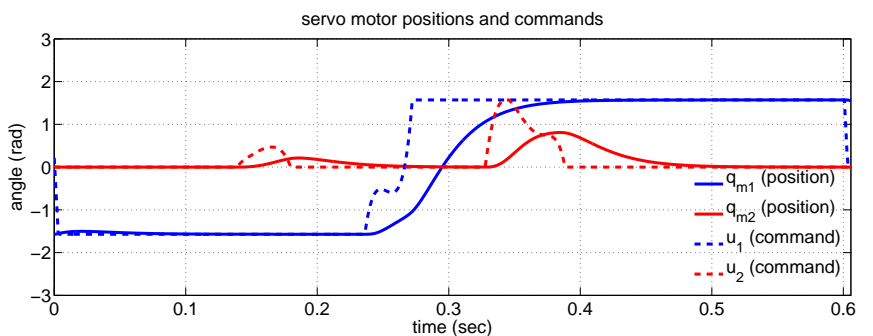

Fig. 3. Servo motor commands $u_{i}$ (dotted line) and actual angle $q_{m i}$ (solid line) for the results with optimal movement duration $T=0.606$ (cf. Fig. 2 (b) bottom). Servo motor response delay can be observed characterized by the servo motor dynamics 8 . The proposed optimal control framework finds appropriate control commands taking this effect into account.

to minimizing the stiffness $k$ in an approximated way. Note that it is possible to directly use $k$ in the cost function. However, in practice, first and second derivatives of $k$ are needed to implement the ILQG algorithm which become significantly more complex than those of $F$ since the joint stiffness $k$ is already the first derivative of $\tau$ as described in (5). Thus, it is preferable to use the spring tension $F$. This close relationship between $F$ and $k$ in the general nonlinear case can be observed in the plots, for example, in Fig. 2 (middle and bottom in (c)). In fact, the appropriate choice of the cost function is critical for successful task execution. We discuss the issue of task encoding via cost selection in Section IV-D in more details.

1) Swing Locomotion: Consider the case where that target bar is located at $d=0.3(\mathrm{~m})$. We optimize both the control command $\mathbf{u}$ and the movement duration $T$. We use $\mathbf{Q}_{T}=$ $\operatorname{diag}\{10000,10000,10,10\}, \mathbf{R}_{1}=\operatorname{diag}\{0.0001,0.0001\}$ and $R_{2}=0.1$ for the cost function in (19). As mentioned above, $\mathbf{R}_{1}$ is chosen to be a small value for regularization needed for ILQG implementation. The optimized movement duration was $T=0.606$ (sec).

Fig. 2 shows (a) the optimized robot movement, (b) joint trajectories and servo motor positions, and (c) joint torque, spring tension and joint stiffness. In the plots, trajectories of the fixed time horizon ranging $T=0.5 \sim 0.7$ (sec) are also overlayed for comparison in addition to the case of the optimal movement duration $T=0.606$ ( $\mathrm{sec}$ ). In the optimized movement, the spring tension and the joint stiffness are kept small at the beginning and end of the movement resulting in nearly zero joint torque, which allows the joint to swing passively. The joint torque is exerted only during the middle of the swing by increasing the spring tension as necessary. This result suggests that the natural plant dynamics are fully exploited for the desirable task execution based on the control strategy discussed in Section II with simultaneous stiffness and temporal optimization.

In order to illustrate the effect of the servo motor dynamics characterized by (8), Fig. 3 shows the servo motor position commands and actual motor angles with the optimal movement duration (cf. Fig. 22 (b) bottom). Delays in the servo motor response can be observed in this plot. This suggests that the proposed optimal control framework can find appropriate control commands taking this effect into account.



Fig. 4. Effect of optimal feedback under the presence of parameter mismatch between the nominal model used to compute optimal control and the actual robot. Solid line: the movement with the optimal feedback control. Red line: the movement with only feedforward command. The result demonstrates the effectiveness of optimal feedback control under model uncertainty.

\section{B. Effect of Optimal Feedback under Modelling Error}

One of the benefits of using the optimal feedback control framework is that in addition to computing the optimal feedforward control command, it provides a locally optimal feedback control in the neighborhood of the optimal trajectory, which allows the controller to make corrections if there is small deviation from the nominal optimal trajectory. In this section, we present numerical studies of the effect of optimal feedback control (14) under the influence of model mismatch between the nominal model and actual robot parameters. We introduce a modelling error as $m_{i, \text { nominal }}=1.05 m_{i}$ (link mass) and $l_{c, i, \text { nominal }}=1.1 l_{c, i}$ (location of center of mass on the link) for $i=1,2$.

Fig. 4 shows the comparison between the movement using the optimal feedback control law (14) obtained in the simulation in Section IV-A1 above and with only feedforward (open loop) optimal control command $\mathbf{u}=\mathbf{u}^{\text {opt }}(t)$ under the presence of modelling error. Using only feedforward control, the robot deviates from the target bar due to the model mismatch. However, with the optimal feedback control law, the robot is able to get closer towards the target with the help of the feedback term. These results suggest the effectiveness of the optimal feedback control. In future work, we are interested in on-line learning of the plant dynamics to address the issue of model uncertainty [20, 21].

\section{Switching Dynamics and Tasks Parameters}

In this section, we explore different task requirements with switching dynamics. In the following simulation, we use the robot model with the link length as $l_{1}=0.2(\mathrm{~m})$ and $l_{2}=0.35(\mathrm{~m})$ introducing asymmetric configuration in the robot structure. We consider the task of first swinging up from the suspended posture to the target at $d=0.45(\mathrm{~m})$, then subsequently continuing to locomote twice to the target bars at $d=0.4(\mathrm{~m})$ and $d=0.42(\mathrm{~m})$ (irregular intervals). Note that every time the robot grasps the target and starts swinging for the next target, the robot configuration is interchanged, which significantly changes the dynamic properties for each swing movement due to asymmetric structure of the robot. Thus, the stiffness and movement duration need to be adjusted 


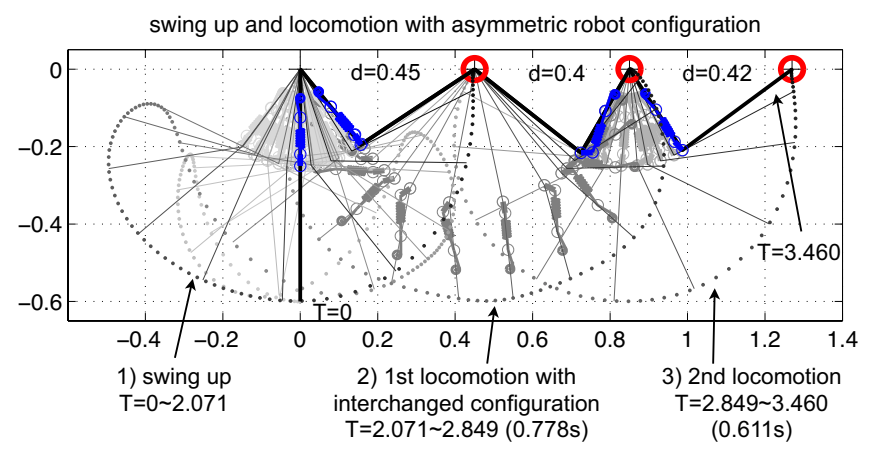

(a) Sequence of the movement of the robot

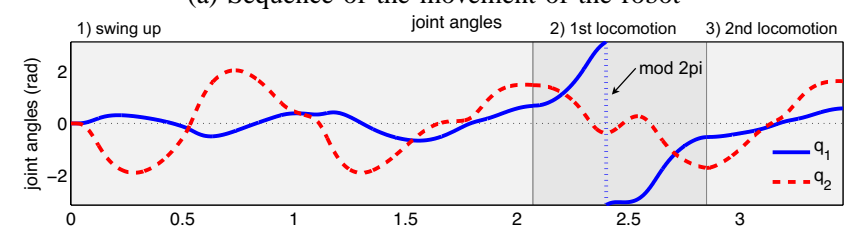

servo motor positions

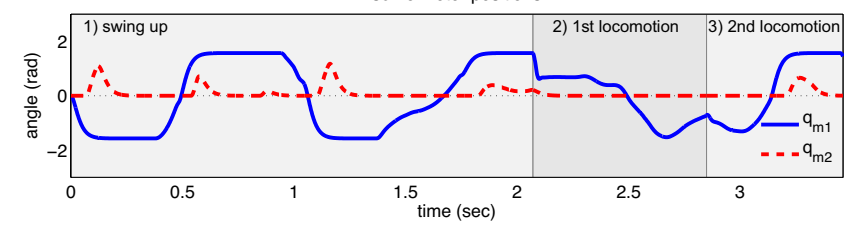

(b) Joint trajectories and servo motor positions
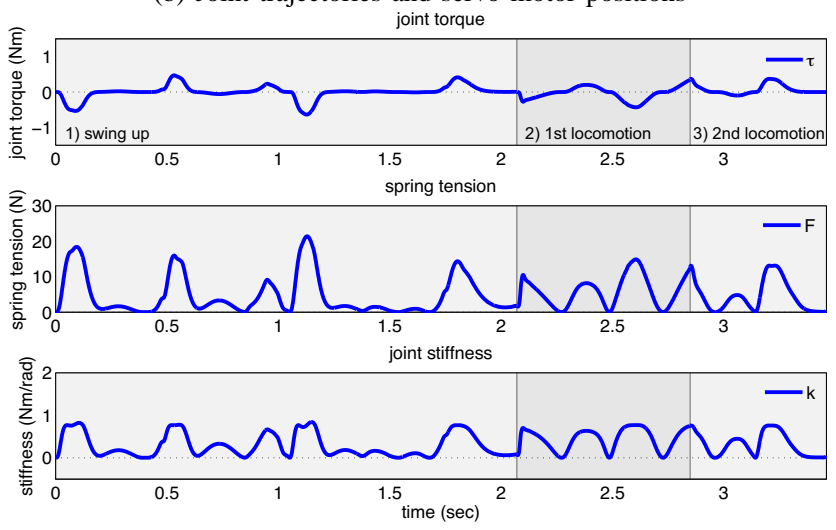

(c) Joint torque, spring tension and joint stiffness

Fig. 5. Simulation results of the sequence of movements. Note that the robot configuration is asymmetric with the link length $l_{1}=0.2(\mathrm{~m})$ and $l_{2}=0.35$ (m). When the robot swings after grasping the bar, the robot configuration is interchanged, which significantly changes the dynamic characteristics.

appropriately to fulfill the desired task requirement. The cost function (19) with the same parameters are used as in the previous simulations. For the swing-up task, we add the time cost $w_{T} T$ with $w_{T}=5$ (see (18)), i.e., the task requirement in swing-up is try to swing up quickly while minimizing the control cost.

Fig. 5 shows (a) the sequence of the optimized robot movement, (b) joint trajectories and servo motor positions, and (c) joint torque, spring tension and joint stiffness. The obtained optimal movement duration was The obtained optimal movement duration was 1) $T=2.071$ (sec) for swing up, 2) $T=0.778$ (sec) for the locomotion with interchanged (upside down) robot model and 3) $T=0.611$ (sec) for the last swing movement, respectively. Notice the significant difference in the optimized movement time for the swing locomotion 2) and 3) (more than 25\%). In 2), the lower link is heavier and in 3) the top link is heavier due to the mass of the VSA model. Thus, the effective natural frequency of the pendulum movement is different, which resulted in different movement duration. The results highlight that our approach can find appropriate movement duration and command sequence to achieve the task under different requirement and conditions (locomotion, swing-up, robot dynamics change and target distance change). In this example, each maneuver is optimized separately. Optimization over multiple swing movements including transitions will be of our future interest.

\section{Design and Selection of a Cost Function}

In optimal control, generally, a task is encoded in terms of a cost function, which can be viewed as an abstract representation of the task. From our point of view and experience, design and selection of the cost function is one of the most important and difficult parts for a successful application of such an optimal control framework. For a simple task and plant dynamics, an intuitive choice (typically a quadratic cost in the state and control as in an LQR setting) would suffice (still it is necessary to adjust the weights). However, for a highly dynamic task with complex plant dynamics, this increasingly becomes difficult and an appropriate choice of the cost function which best encodes the task still remains an open issue.

In this section, we explore a few more candidates of the cost functions. In addition to the cost function (19), consider the following running cost functions $h=h(\mathbf{x}, \mathbf{u})$ in (13):

- quadratic cost with the control command (servo motor position command):

$$
h=\mathbf{u}^{T} \mathbf{R u}
$$

- quadratic cost with the joint torque. The main term is the cost associated with the joint torque $\tau$ and $\mathbf{u}^{T} \mathbf{R}_{1} \mathbf{u}$ is added for regularization (small $\mathbf{R}_{1}$ ):

$$
h=\mathbf{u}^{T} \mathbf{R}_{1} \mathbf{u}+R_{2} \tau^{2}
$$

Figure 6 shows the results using the running cost $h=$ $\mathbf{u}^{T} \mathbf{R u}$ in (23) with $\mathbf{R}=\operatorname{diag}\{1,1\}$. The obtained optimal movement duration is $T=0.604(\mathrm{sec})$. Figure 7 shows the results using the running cost $h=\mathbf{u}^{T} \mathbf{R}_{1} \mathbf{u}+R_{2} \tau^{2}$ in (24) with $\mathbf{R}_{1}=\operatorname{diag}\{0.0001,0.0001\}$ and $R_{2}=100$. The obtained optimal movement duration is $T=0.620$ (sec). In both of these two cases, the same terminal cost parameters are used as in the case of (19).

As demonstrated in Figs. 6 and 7 the robot is also successfully able to reach the target bar by minimizing each specific cost in addition to the case of the cost (19) presented in Section IV-A above. However, with the choice of the running cost (23), significant difference in the resultant robot movement and much higher spring tension and joint stiffness can be observed in Fig. 6 As can be seen in Fig. 7 , with the choice of cost associated with the joint torque in 24), the resultant movement looks almost identical to the one with the cost (19) (see Fig. 2) and the joint torque profile is comparable. However, we can 

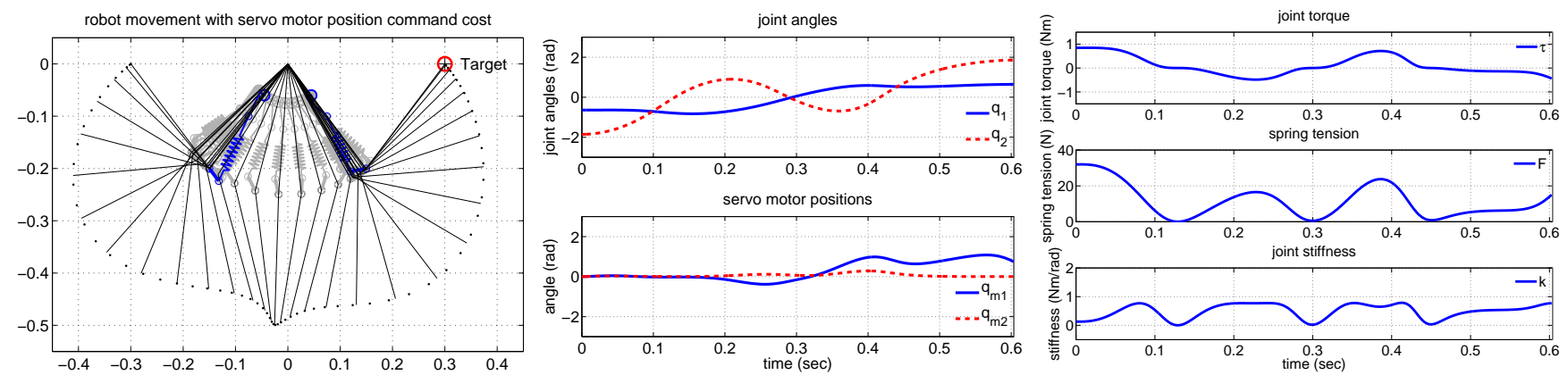

Fig. 6. Optimization of the locomotion task using the running cost $l=\mathbf{u}^{T} \mathbf{R}_{1} \mathbf{u}$ in 23 with $\mathbf{R}_{1}=\operatorname{diag}\{1,1\}$. Left: Movement of the robot with optimized duration $T=0.604(\mathrm{sec})$ Center: Joint angles and servo motor angles. Right: Joint torque, spring tension and joint stiffness. Note that while the task itself is achieved, the movement looks very different from the one in Fig. 2 and much higher spring tension and joint stiffness during the swing movement can be observed.


Fig. 7. Optimization of the locomotion task using the running cost $l=\mathbf{u}^{T} \mathbf{R}_{1} \mathbf{u}+R_{2} \tau^{2}$ in 24 with $\mathbf{R}_{1}=\operatorname{diag}\{0.0001,0.0001\}$ and $R_{2}=100$. Left: Movement of the robot with optimized duration $T=0.620(\mathrm{sec})$ Center: Joint angles and servo motor angles. Right: Joint torque, spring tension and joint stiffness. The resultant movement looks almost identical to the one in Fig. 2 and the joint torque profile is comparable. However, we can observe that spring tension and joint stiffness are larger than those of Fig. 2

observe that spring tension and joint stiffness are larger than those of the cost (19). This is due to the redundancy in the variable stiffness actuation and the results depend on how we resolve it by an appropriate choice of the cost function. These results suggest that the choice of the cost function is crucial, however, its selection is still non-intuitive.

Note that other consideration of cost functions could be possible, e.g., energy consumption. In the brachiation task, without friction, the mechanical energy of the rigid body dynamics, $E=\int_{0}^{T} \tau \dot{q}_{2} d t$, is conserved for the swing locomotion with the same intervals at the same height starting and ending at zero velocity (if no potential energy is stored in the spring of the VSA at the end of the swing). Thus, if we wish to consider true energy consumption, it would be necessary to evaluate the electrical energy consumed at the motor level. However, this is not straightforward since we need a precise model of the mechanical and electrical motor dynamics including all the factors such as motor efficiency and transmission loss, which could be rather complex to model in practice, and the control strategy would largely depend on the properties of the actual motors used.

\section{E. Remarks on other Joint Actuation and Controller Design Approaches}

In this paper, we explore variable stiffness actuation to exploit passive dynamics in swing movement. One of the desirable properties of the variable stiffness actuation we consider is that the joint can be fully mechanically passive by appropriately adjusting the spring tension in the actuator mechanisms. This is in contrast to joint actuation with geared electric motors in many of existing robotic systems. Typically, joints with geared motors with high gear ratio aimed for position control cannot be fully back-drivable, i.e., joints cannot be made passive to exploit natural dynamics of the link. For example, the brachiating robot in [23] uses a DC motor with a harmonic drive gear and exhibited complex and relatively high friction. Thus, in this design, it is not possible to exploit the passive dynamics of the second link since the motor is not fully back-drivable by gravity, and it is necessary to actively drive the joint to achieve the swing movement. To make the joint fully back-drivable without passive components, we may need to use high performance direct drive motors which would typically require precise torque control mechanisms.

From the viewpoint of a different controller design approach, the target dynamics method [23] uses input-output linearization to actively cancel the plant dynamics. While its effectiveness has been demonstrated in the torque controlled robot hardware, it is not straightforward to apply this method to the control of robot with general variable stiffness mechanisms since the system dynamics are not easily input-output linearizable due to redundancy and complex nonlinearity in actuator dynamics. Furthermore, it turned out that for the parameter setting used in Section IV-C, the target dynamics controller becomes singular at some joint angle $q_{2}$ within the range of the movement even for the torque controlled case. With the link mass parameters used in this paper, we did not find problems with the same link length $l_{1}=l_{2}$, however, typically, we numerically found that when $l_{2}>l_{1}$, the 
target dynamics method encounters an ill-posedness problem of invertibility in the derivation of the control law (cf. Equation (15) in [23]).

\section{CONCLUSION}

In this paper, we have presented an optimal control framework for exploiting passive dynamics of the system for swing movements. As an example, we considered brachiation on a two-link underactuated robot with a variable stiffness actuation mechanism, which is a highly dynamic and challenging task. Numerical simulations illustrated that our framework was able to simultaneously optimize the time-varying joint stiffness profile and the movement duration exploiting the passive dynamics of the system. These results demonstrate that our approach can deal with different task requirements (locomotion in different intervals, swing-up), modelling errors and switching in the robot dynamics. In addition, we empirically explored the issue of the design and selection of an appropriate cost function for successful task execution.

The approach presented in this paper to exploit the passive dynamics with VSA contrasts to the nonlinear controller design with active cancellation of the plant dynamics using input-output linearization for the same task [23]. However, we feel that it shares an important issue of task encoding (or description) either in the form of target dynamics or in terms of a cost function based on physical understanding and insight into the task. We aim to extend our approach to include variable damping [26] for dynamic tasks involving interactions with environments.

\section{ACKNOWLEDGMENTS}

This work was funded by the EU Seventh Framework Programme (FP7) as part of the STIFF project.

\section{REFERENCES}

[1] K. Akawaza, et al. Modulation of stretch reflexes during locomotion in the mesencephalic cat. J. of Physiolosy, 329 (1):553-567, 1982.

[2] D. J. Bennett, et al. Time-varying stiffness of human elbow joint during cyclic voluntary movement. Exp. Brain Res., 88: 433-442, 1992.

[3] D. Braun, M. Howard, and S. Vijayakumar. Optimal Variable Stiffness Control: Formulation and Application to Explosive Movement Tasks. Autonomous Robots, 2012. (in press).

[4] M. G. Catalano, R. Schiavi, and A. Bicchi. Mechanism design for variable stiffness actuation based on enumeration and analysis of performance. In IEEE ICRA, 2010.

[5] S. Collins, A. Ruina, R. Tedrake, and M. Wisse. Efficient bipedal robots based on passive-dynamic walkers. Science, 307 (5712):1082-1085, 2005.

[6] O. Eiberger, et al. On joint design with intrinsic variable compliance: derivation of the DLR QA-joint. In IEEE ICRA, 2010.

[7] S. Eimerl and I. DeVore. The Primates. TIME-LIFE BOOKS, 1966.

[8] M. Garabini, A. Passaglia, F. Belo, P. Salaris, and A. Bicchi. Optimality principles in variable stiffness control: The VSA hammer. In IEEE/RSJ IROS, 2011.

[9] M. W. Gomes and A. L. Ruina. A five-link 2D brachiating ape model with life-like zero-energy-cost motions. J. of Theoretical Biology, 237(3):265-278, 2005.
[10] S. Haddadin, M. Weis, S. Wolf, and A. Albu-Schaëffer. Optimal control for maximizing link velocity of robotic variable stiffness joints. In IFAC World Congress, 2011.

[11] R. Van Ham, et al. MACCEPA, the mechanically adjustable compliance and controllable equilibrium position actuator: Design and implementation in a biped robot. Rob. and Aut. Sys., 55(10):761-768, 2007.

[12] R. Van Ham, et al. Compliant actuator designs. IEEE Robotics and Automation Mag., 16(3):81-94, 2009.

[13] T. Hondo and I. Mizuuchi. Analysis of the 1-joint springmotor coupling system and optimization criteria focusing on the velocity increasing effect. In IEEE ICRA, 2011.

[14] J. W. Hurst, J. E. Chestnutt, and A. A. Rizzi. The actuator with mechanically adjustable series compliance. IEEE Trans. on Robotics, 26(4):597-606, 2010.

[15] A. Jafari, N. G. Tsagarakis, B. Vanderborght, and D. G. Caldwell. A novel actuator with adjustable stiffness (AwAS). In IEEE/RSJ IROS, 2010.

[16] J. G. D. Karssen and M. Wisse. Running with improved disturbance rejection by using non-linear leg springs. Int. J. of Robotics Research, 30(13):1585-15956, 2011.

[17] W. Li and E. Todorov. Iterative linearization methods for approximately optimal control and estimation of non-linear stochastic system. Int. J. of Control, 80(9):1439-1453, 2007.

[18] A. De Luca and G. Oriolo. Trajectory planning and control for planar robots with passive last joint. Int. J. of Robotics Research, 21(5-6):575-590, 2002.

[19] K. M. Lynch, et al. Collision-free trajectory planning for a 3DOF robot with a passive joint. Int. J. of Robotics Research, 19(12):1171-1184, 2000.

[20] D. Mitrovic, S. Klanke, R. Osu, M. Kawato, and S. Vijayakumar. A computational model of limb impedance control based on principles of internal model uncertainty. PLOS ONE, 2010.

[21] D. Mitrovic, S. Klanke, and S. Vijayakumar. Learning Impedance Control of Antagonistic Systems Based on Stochastic Optimization Principles. Int. J. of Robotics Research, 2010.

[22] Y. Nakamura, et al. Nonlinear behavior and control of a nonholonomic free-joint manipulator. IEEE Trans. on Rob. and Aut., 13(6):853-862, 1997.

[23] J. Nakanishi, T. Fukuda, and D. E. Koditschek. A brachiating robot controller. IEEE Trans. on Robotics and Automation, 16 (2):109-123, 2000.

[24] J. Nakanishi, K. Rawlik, and S. Vijayakumar. Stiffness and temporal optimization in periodic movements: An optimal control approach. In IEEE/RSJ IROS, 2011.

[25] G. Palli, C. Melchiorri, and A. De Luca. On the feedback linearization of robots with variable joint stiffness. In IEEE ICRA, 2008.

[26] A. Radulescu, M. Howard, D. J. Braun, and S. Vijayakumar. Exploiting variable physical damping in rapid movement tasks. In IEEE/ASME AIM Conf., 2012.

[27] K. Rawlik, M. Toussaint, and S. Vijayakumar. An approximate inference approach to temporal optimization in optimal control. NIPS 2010.

[28] N. Rosa, A. Barber, R. D. Gregg, and K. Lynch. Stable openloop brachiation on a vertical wall. In IEEE ICRA, 2012.

[29] F. Saito, T. Fukuda, and F. Arai. Swing and locomotion control for a two-link brachiation robot. IEEE Cont. Sys. Mag., 14(1): 5-12, 1994.

[30] R. Shadmehr. Learning virtual equilibrium trajectories for control of a robot arm. Neural Computation, 2(4):436-446, 1990.

[31] M. W. Spong. Modeling and control of elastic joint robots. Trans. of ASME, J. of Dynamic Systems, Measurement, and Control, 109(6):310-319, 1987.

[32] M. W. Spong. The swing up control problem for the acrobot. IEEE Control Sys. Mag., 15(1):49-55, 1995

[33] R. F. Stengel. Optimal Control and Estimation. 1994. 